\title{
Linear Viscoelasticity of Emulsions
}

\author{
II. Measurements of the Linear Viscoelastic Behavior of Emulsions \\ in the Kilohertz Range
}

\author{
M. OOSTERBROEK, ${ }^{1} \mathrm{~J}$. MELLEMA, AND J. S. LOPULISSA \\ Department of Applied Physics, Twente University of Technology, Enschede, The Netherlands
}

Received August 14, 1980; accepted March 10, 1981

\begin{abstract}
Linear viscoelasticity of emulsions in shear deformation in the kilohertz range is demonstrated experimentally. In order to avoid complications due to inertia effects, emulsions with small droplet sizes are studied. The preliminary measurements are interpreted as being the result of droplet deformations. If this interpretation is correct, measurements of the dynamic viscosity of emulsions may be used to obtain information about the mechanical properties of the interfacial layer between droplets and the continuous phase. In particular, the evaluation of the interfacial tension of emulsion droplets from bulk properties might be possible using this technique.
\end{abstract}

\section{INTRODUCTION}

In a previous paper (1) two distinct models for describing the linear viscoelasticity of nondilute emulsions were presented. In these models the viscoelasticity is due to the deformation of droplets of a Newtonian fluid immersed in another Newtonian fluid.

The models predict two or more relaxation times. One of these, e.g., $\lambda_{y}$, may be related to the interfacial tension: $\lambda_{\gamma} \approx a \eta / \gamma$. The pertaining relaxation mechanism will affect $\eta_{1}$ and $\eta_{2}$ at frequencies $\nu$ around $\nu_{\gamma}$ $=\left(2 \pi \lambda_{\gamma}\right)^{-1}$. For microemulsions $\left(\gamma=10^{-2}\right.$ $\mathrm{mN} \mathrm{m} \mathrm{m}^{-1} ; a \simeq 30 \mathrm{~nm} ; \eta \simeq 1 \mathrm{mPa} \mathrm{sec}$ ) this frequency lies in the kilohertz range.

Recent progress in measuring techniques $(2,3)$ enables us to determine the dynamic viscosity of liquids relative to that of a calibration liquid between 4 and $200 \mathrm{kHz}$ with an error of less than 0.02 . The theory mentioned above predicts significant changes in the dynamic viscosity of emulsions if the frequency is varied around $\nu_{\gamma}$. These changes

\footnotetext{
${ }^{1}$ Present address: Akzo Corporate Research, Arnhem, The Netherlands.
}

will become detectable at particle concentrations above about 0.05 by volume. At these concentrations, however, particleparticle interactions can no longer be disregarded, and a theory that also covers the case of nondilute emulsions will be needed for interpretation of the measurement.

Another restrictive condition for the measurements arises from inertia effects. We shall not go into detail here, as it is sufficient to say that the rather complicated effects may completely obscure the relation between droplet properties (interfacial tension and interfacial rheology) and the response of the system to a measuring probe. These effects remain unimportant (4) as long as the condition $a^{2} \omega \rho / \eta \ll 1$ is satisfied. This condition no longer holds at frequencies above $10 \mathrm{kHz}$ for droplet radii of about $1 \mu \mathrm{m}$.

The aim of our investigations is to demonstrate the linear viscoelasticity of emulsions. Because of the inertia effects just mentioned we will confine ourselves to emulsions with very small droplets. The preliminary results will be interpreted as effects due to droplet deformations. 
TABLE I

Mesoemulsion Properties at $20.0^{\circ} \mathrm{C}$

\begin{tabular}{|c|c|c|c|c|c|c|c|c|}
\hline \multirow[b]{2}{*}{ System } & \multirow[b]{2}{*}{ Dilution } & $\phi_{\mathrm{B}}$ & $\begin{array}{c}c \\
\left(\mathrm{~g} / \mathrm{cm}^{s}\right)\end{array}$ & $\phi_{\mathrm{B}}$ & $\begin{array}{c}c \\
\left(\mathrm{~g} / \mathrm{cm}^{3}\right)\end{array}$ & & $a$ & \\
\hline & & \multicolumn{2}{|c|}{ as weighed in } & \multicolumn{2}{|c|}{ experimental } & $\phi$ & $(\mathrm{nm})$ & $\bar{\eta} / \eta_{0}$ \\
\hline ms 1 & None & 0.173 & 0.050 & 0.169 & 0.050 & 0.20 & $30 \pm 10$ & 1.99 \\
\hline $\mathrm{ms} 2 \mathrm{~A}$ & None & 0.200 & 0.048 & 0.188 & 0.048 & 0.21 & $50 \pm 10$ & 2.15 \\
\hline $\mathrm{ms} 2 \mathrm{~B}$ & $3: 1$ & 0.150 & 0.043 & 0.140 & 0.042 & 0.16 & - & 1.77 \\
\hline $\mathrm{ms} 2 \mathrm{C}$ & $1: 1$ & 0.100 & 0.038 & 0.092 & 0.037 & 0.10 & - & 1.36 \\
\hline $\mathrm{ms} 2 \mathrm{D}$ & $1: 3$ & 0.050 & 0.033 & 0.052 & 0.032 & 0.06 & - & 1.19 \\
\hline $\mathrm{ms} 3 \mathrm{~A}$ & None & 0.200 & 0.048 & 0.187 & 0.049 & 0.21 & $50 \pm 10$ & 2.27 \\
\hline $\mathrm{ms} 3 \mathrm{~B}$ & $3: 1$ & 0.150 & 0.043 & 0.141 & - & $0.16^{a}$ & - & 1.69 \\
\hline $\mathrm{ms} \mathrm{3C}$ & $1: 1$ & 0.100 & 0.038 & 0.091 & - & $0.10^{a}$ & - & 1.34 \\
\hline
\end{tabular}

${ }^{a}$ Using $c$ as weighed in.

\section{MATERIALS AND METHODS}

The surfactants used were commercial products. Tween 20 (polyoxyethylene (20) sorbitan monolaurate) was obtained from Atlas Chemie, Essen, Germany. NPE (polyoxyethylated nonyl phenolether) and DPE (polyoxyethylated dodecyl phenolether) with various polyoxyethylene chain lengths were obtained by courtesy of Servo Chemical Co., Delden, The Netherlands. Analytical grade benzene, hexane or cyclohexane was used for the oil phase. Water was treated in a Millipore filter, type "milli-Q2," resulting in an electrical conductivity of 80 $\mu \Omega^{-1} \mathrm{~m}^{-1}$ and an organic carbon content of about 2 ppm.

Steady-state viscosities were measured in a Couette-viscosimeter, type LS 30, manufactured by Contraves, Zürich. All systems that were investigated showed Newtonian behavior at rates of shear between $10^{-2}$ and $10^{2} \mathrm{sec}^{-1}$.

The measurements of linear viscoelastic properties in the frequency range 4-200 $\mathrm{kHz}$ were performed using a torsional nickel tube apparatus $(2,3)$. In this apparatus a premagnetized nickel tube is set into a torsional motion magnetostrictively. The effects of a liquid which surrounds the tube are a change in position and an increase of the band-width of the torsion-resonance peaks. The liquid's dynamic viscosity can be determined from these effects. The measuring procedure was changed so far as that the fundamental frequency $(3.7 \mathrm{kHz})$ was also included in the measurements. The linearity of the viscoelastic properties was checked by performing measurements using different amplitudes of the torsion motion of the tube.

Two types of emulsions were studied. We shall call them mesoemulsions and microemulsions. The mesoemulsions had small droplets $(a \leq 50 \mathrm{~nm})$, did not form spontaneously, and were stable over a limited period of time (months). The microemulsions also had small droplets, but they formed spontaneously if a sample having a suitable composition was stored at the right temperature. Microemulsions are thermodynamically stable, but it may take them some weeks to reach equilibrium. We shall now describe the preparation of two types of emulsions.

The mesoemulsions $\mathrm{ms} 1, \mathrm{~ms} 2 \mathrm{~A}$, and $\mathrm{ms}$ $3 \mathrm{~A}$ were prepared from benzene and a solution of $6.00 \mathrm{~g} / \mathrm{dl}$ Tween 20 in water. Ms 1 was prepared separately, but ms $2 \mathrm{~A}$ and $\mathrm{ms}$ $3 \mathrm{~A}$ were prepared in exactly the same manner, both mechanically and thermally, and at the same time. The components of each emulsion (see Table 1) were kept at $65^{\circ} \mathrm{C}$ in sealed bottles, and then vigorously shaken at that temperature. Next the emulsions obtained were quickly cooled down to $20^{\circ} \mathrm{C}$. 
Finally fractions of $\mathrm{ms} 2 \mathrm{~A}$ and $\mathrm{ms} 3 \mathrm{~A}$ were diluted with a $2.8 \mathrm{~g} / \mathrm{dl}$ solution of Tween 20 in water. This solution had properties close to those of the continuous phase (see next paragraphs). The viscous and dielectrical properties of similar emulsions were described by Matsumoto and Sherman (5) and Clause et al. (6). Since not all of the benzene was taken up in the emulsion, the volume fraction of benzene $\phi_{\mathrm{B}}$ in the mesoemulsions was calculated from density measurements. In this calculation, changes of the partial specific volume of surfactant due to adsorption at the oil-water interface were neglected. The density measurements were performed using a DMA 40 digital densitometer, manufactured by Anton Paar K.G., Graz,Austria.

A part of the continuous phase was separated from the mesoemulsions by the use of a Labofuge 1 centrifuge, manufactured by Heraeus, Osterode am Harz, Germany.

The effective centripetal acceleration was $21 \times 10^{3} \mathrm{msec}^{-2}$ at a speed of $4000 \mathrm{rpm}$. The benzene concentration in the continuous phase was determined by using a Varian gas chromatograph, type 2860 , with a flame ionization detector. The amount of benzene in the continuous phase turned out to be negligible (less than 5 ppm by weight).

Surfactant concentrations were determined from dry-weight analyses. The concentrations in the continuous phases of $\mathrm{ms}$ $1, \mathrm{~ms} 2 \mathrm{~A}$, and $\mathrm{ms} 3 \mathrm{~A}$ appeared to be the same, $c=2.8 \pm 0.2 \mathrm{~g} / \mathrm{dl}$. The density and the

TABLE II

Microemulsion Composition in Volume Fractions

\begin{tabular}{|c|c|c|c|}
\hline System & Oil & Surfactant & $T\left({ }^{\circ} \mathrm{C}\right)$ \\
\hline me 1 & $14 \%$ hexane & $\begin{array}{l}4 \% \text { NPE } 1.5 \\
+4 \% \text { NPE } 15\end{array}$ & 20.0 \\
\hline $\mathrm{mc} 2$ & $24 \%$ cyclohexane & 3\% DPE 8.5 & 24.0 \\
\hline $\operatorname{mc} 3$ & $15 \%$ cyclohexane & $5 \%$ NPE $7^{a}$ & $15.0,16.0$ \\
\hline
\end{tabular}

a For this experiment NPE was purified from free polyethyleneglycol by extraction with ethylacetate (Weibullmethod).

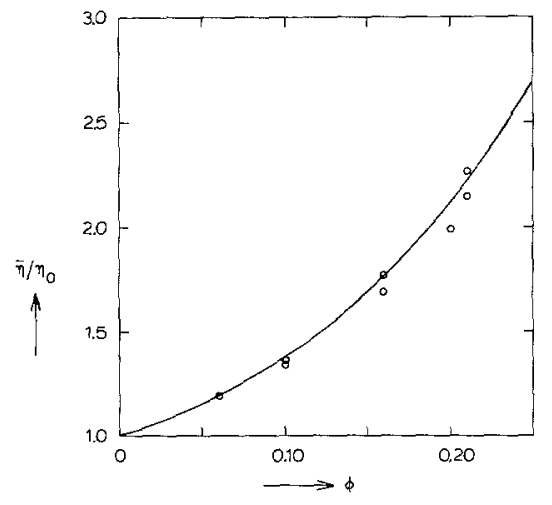

FIG. 1. Relative steady-state viscosity of the mesoemulsions as a function of the particle concentration. The line corresponds with Eq. [2].

steady-state viscosity of the continuous phases were equal to those of a $2.8 \mathrm{~g} / \mathrm{dl}$ Tween 20 in water solution.

The average droplet radius was estimated by using the sedimentation coefficient that was found in the centrifuge runs. The equation used has been derived by Burgers (7):

$$
a=\left(\frac{9 \eta_{0}(1+6.875 \phi) S}{2\left(\rho_{\mathrm{D}}-\rho_{0}\right)}\right)^{1 / 2} \text {. }
$$

In Table I some of the properties of the emulsions are summarized. The total particle volume fraction $\phi$ is the sum of the volume fraction of the dispersed benzene and that of the surfactant adsorbed at the droplet surfaces. In the calculation the specific volume of the adsorbed surfactant is assumed to be the same as that of the surfactant in the continuous phase. Furthermore, it is assumed that the droplets contain no water. If the latter assumption is justified the uncertainty in $\phi$ is about 0.005 .

The microemulsions were prepared from hexane or cyclohexane, water, and NPE or DPE surfactants. The phase behavior of similar systems was studied by Shinoda and Kunieda (8).

Series of samples were made containing fixed amounts of oil, water, and surfactant, while the average length of the surfactant's polyoxyethylene chain was varied. The components of each sample were mixed by 


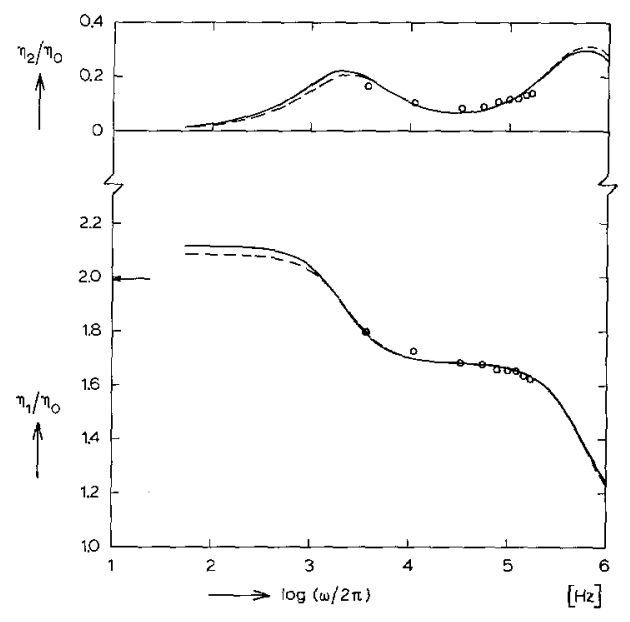

FIG. 2. Relative dynamic viscosity of the mesoemulsion $\mathrm{ms} 1$ as a function of frequency at $20.0^{\circ} \mathrm{C}$. Measurements indicated with open circles. Line, model A; dashes, model $\mathrm{B}$. The arrow on the left marks the steady-state viscosity.

being shaken together in a sealed bottle. The samples were then left undisturbed at a constant temperature for 2 weeks. The formation of microemulsions was recognized from the translucency of the one-phase systems obtained. The composition of three typical microemulsions is given in Table II. Microemulsions containing only nonionic surfactants are very sensitive to changes in temperature. Droplet sizes, compositions of the continuous phase, and steady-state viscosities at various rates of shear were therefore hard to determine for these systems, and are not included in this preliminary investigation.

\section{RESULTS}

In Fig. 1 the relative steady-state viscosity of the mesoemulsions as a function of the particle concentration is compared with Simha's (9) theoretical expression for the viscosity of a suspension of rigid spheres:

$$
\begin{aligned}
& \bar{\eta} / \eta_{0}=1+10 \phi R^{3}\left(R^{7}-1\right) / \\
& \quad\left\{4\left(R^{10}+1\right)-25\left(R^{7}+R^{3}\right)+45 R^{5}\right\},
\end{aligned}
$$

where $R=f \phi^{-1 / 3}$. For the correction fac- tor we have taken the value $f=1.111$, this being the value Thomas (10) found on analyzing data for suspensions of rigid spheres at high shear rates and at low concentrations $(\phi<0.1)$. The agreement of this analysis with our data on mesoemulsions is good even at higher concentrations.

This agreement need not surprise us, although emulsion droplets are in principle deformable and our measurements were performed at relatively low rates of shear. In Ref. (1) we demonstrated that droplets which possess a surface elasticity behave like rigid spheres at low frequency. The influence of the shear rate on the viscosity of a suspension was investigated by Krieger (11). He established a rheological equation of state for suspensions of rigid spheres. This equation describes the transition from a low-shear viscosity to a high-shear viscosity. The transition is caused by the interplay between the Brownian forces and the shear forces on the particles. The magnitude of the transition in the relative viscosity increases with the particle concentration. For $\phi$ $<0.20$ the difference between the relative viscosities in the high and low shear rate limit, as interpolated from Krieger's results, is comparable with the experimental errors in our steady-state viscosity measurements.

In Fig. 2 the dynamic viscosity of $\mathrm{ms} 1$ relative to that of the $2.8 \mathrm{~g} / \mathrm{dl}$ Tween 20 in water solution is shown as a function of the frequency. (Measurements of the dynamic viscosity of the $2.8 \mathrm{~g} / \mathrm{dl}$ Tween 20 in water solution are not given here, since the solution behaves completely Newtonian up to the highest frequency attainable with our instrument.) As the error in the measurements of $\eta_{2} / \eta_{0}$ is about 0.02 , and $\eta_{2} / \eta_{0}$ should vanish for $\omega \rightarrow 0$ and $\omega \rightarrow \infty$, it may be concluded that $\eta_{2}$ in Fig. 2 has at least two maxima. The steady-state value of $\eta_{1} / \eta_{0}$ is larger than the measured dynamic viscosity $\eta_{1} / \eta_{0}$ at the lowest frequency. At the highest frequencies the measured $\eta_{1} / \eta_{0}$ values are decreasing for increasing $\omega$. This suggests that the limit value of $\eta_{1} / \eta_{0}$ for $\omega \rightarrow \infty$ is 
TABLE III

Curve Fit Parameters

\begin{tabular}{|c|c|c|c|c|}
\hline Sample & \multicolumn{2}{|c|}{$\mathrm{ms} 1$} & \multicolumn{2}{|c|}{ ms 2} \\
\hline Experimental data & \multicolumn{2}{|c|}{$\begin{aligned} a & =30 \mathrm{~nm} \\
\eta_{0} & =1.25 \mathrm{mPa} \cdot \mathrm{sec} \\
\eta^{\prime} & =0.65 \mathrm{mPa} \cdot \mathrm{sec} \\
\phi & =0.20\end{aligned}$} & \multicolumn{2}{|c|}{$\begin{aligned} a & =50 \mathrm{~nm} \\
\eta_{0} & =1.25 \mathrm{mPa} \cdot \mathrm{sec} \\
\eta^{\prime} & =0.65 \mathrm{mPa} \cdot \mathrm{sec} \\
\phi & =0.06 ; 0.10 ; 0.16 ; 0.21\end{aligned}$} \\
\hline Model used & A & B & A & B \\
\hline Assumed data & $\begin{array}{l}\mu=0 \\
\sigma=0\end{array}$ & $d=3 \mathrm{~nm}$ & $\begin{array}{l}\mu=0 \\
\sigma=0\end{array}$ & $d=3 \mathrm{~nm}$ \\
\hline Results of fits & $\begin{array}{l}\gamma=0.45 \mathrm{mN} \mathrm{m}^{-1} \\
\kappa=1.1 \mu \mathrm{N} \mathrm{m}^{-1} \\
\zeta=40 \mathrm{pN} \mathrm{sec} \mathrm{m}^{-1}\end{array}$ & $\begin{aligned} \gamma^{\prime} & =0.41 \mathrm{mN} \mathrm{m}^{-1} \\
\gamma & =70 \mu \mathrm{N} \mathrm{m}^{-1} \\
\eta^{\prime \prime} & =7 \mathrm{mPa} \cdot \mathrm{sec}\end{aligned}$ & $\begin{array}{l}\gamma=0.38 \mathrm{mN} \mathrm{m}^{-1} \\
\kappa=0.7 \mu \mathrm{N} \mathrm{m}^{-1} \\
\zeta=100 \mathrm{pN} \mathrm{sec} \mathrm{m}^{-1}\end{array}$ & $\begin{aligned} \gamma^{\prime} & =0.43 \mathrm{mN} \mathrm{m}^{-1} \\
\gamma & =90 \mu \mathrm{N} \mathrm{m}^{-1} \\
\eta^{\prime \prime} & =11 \mathrm{mPa} \cdot \mathrm{sec}\end{aligned}$ \\
\hline
\end{tabular}

lower than the measured $\eta_{1} / \eta_{0}$ value at the highest frequencies. Summarizing we conclude that the experimental data combined with expectations based on general linear viscoelasticity indicate two or more relaxation mechanisms to operate in $\mathrm{ms} 1$. The solid and dashed lines are computer fits for model $A$ and model $B$, respectively (see Ref. (1)).

The comparison of the predictions of these models with the experimental results implies that it is assumed that the observed viscoelasticity is caused by droplet deformation. This assumption is not self-evident and is criticized in the Discussion. It is assumed that the interfacial rheology of model A may be described by a Voigt-Kelvin model. In this case both model A and B contain two relaxation mechanisms. The predicted linear viscoelastic behavior is determined by five constants: two relaxation times, two relaxation strengths, and a steady-state viscosity. The steady-state viscosity of both models is already in agreement with the experimental data as a result of choosing $f=1.111$. An unambiguous fit of the experimental data in terms of the remaining four constants is not feasible, as the frequency range covered by our instrument is not large enough. Consequently further assumptions about the microscopic param- eters are needed in other to reduce the problem to that of a three-parameter curve fitting.

Two classes of fits are possible using model $\mathrm{A}$ : one with $\gamma \gg \mu, \kappa$, and the other one with $\mu \gg \gamma, \kappa$. (A fit where $\kappa \gg \mu, \gamma$ appeared to be incompatible with our experiments.) The fit with $\mu \gg \gamma, \kappa$ required $\gamma<1 \mu \mathrm{N} \mathrm{m}^{-1}$. As this value is probably too small for the mesoemulsion systems, this class of fits was rejected, and only the fits with $\gamma \gg \mu, \kappa$ remained. From Eq. [33] of Ref. (1) it is clear that the larger relaxation time is then a function of $3 \kappa+2 \mu$. In the fits we took $\mu=0$, but any linear combination of $\mu$ and $\kappa$ can be used. Furthermore, the area viscosity $\sigma$ was taken to be zero (see Discussion). The interfacial shear viscosity $\zeta$ was taken as nonzero in order to account for the friction between the surfactant molecules in the interfacial film. If there is no surfactant inside the droplets (e.g., solubilized in benzene) and the droplets are monodisperse, an average area per molecule Tween 20 adsorbed at the droplet surfaces can be estimated from the data of Table I, using the formula

$$
A \simeq \frac{3 \phi M}{a N_{\mathrm{A}}\left(c-c_{0}\right)} .
$$

It follows that $A$ has a minimum value of about $1.5 \mathrm{~nm}^{2} . A$ is even larger when some 


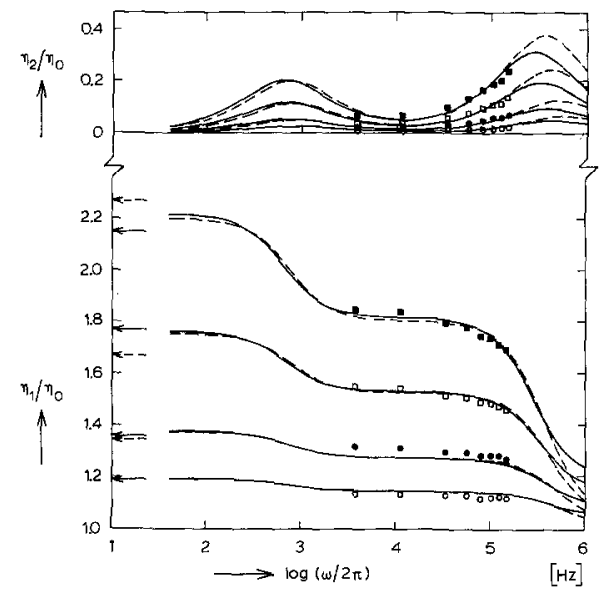

Frg. 3. Relative dynamic viscosity of the mesoemulsion $\mathrm{ms} 2 \mathrm{~A}$, and its dilutions at $20.0^{\circ} \mathrm{C}$ as a function of frequency. Measurements: filled squares, $\phi=0.21$; open squares, $\phi=0.16$; filled circles, $\phi=0.10$; open circles, $\phi=0.06$. Line, model A; dashes, model B. The arrows on the left mark measured steady-state viscosities. Dashed arrows correspond with $\mathrm{ms} 3$.

surfactant is present inside the droplets, which is not unlikely since Tween 20 consists of a blend of surfactants with various polyoxyethylene chain lengths. Since the estimated molecular area is quite large compared to that found for a monomolecular film at an air-water interface $\left(A \approx 0.5 \mathrm{~nm}^{2}\right.$ (12)), we will further consider the interfacial film of our mesoemulsion to be monomolecular. Consequently the value of the film thickness in model B $(d=3 \mathrm{~nm})$ was chosen in agreement with estimations of the average chain length of nonionic surfactants (13).

In the curve fits the measured values of both $\eta_{1}$ and $\eta_{2}$ were used. All points were given the same weight. For both fits the sum of the squares of the deviations between the best fit and the 16 points was $0.4 \times 10^{-2}$. A survey of the experimental, the assumed, and the obtained values of the parameters of the two models is given in Table III.

No deviations beyond the experimental errors were observed between the dynamic viscosities of the emulsions $\mathrm{ms} 2$ and $\mathrm{ms} 3$. The differences observed are difficult to display in a graph. For this reason only the dynamic viscosities of $\mathrm{ms} 2$ relative to a $2.8 \mathrm{~g} / \mathrm{dl}$ solution of Tween 20 in water are shown in Fig. 3.

The difference between the steady-state viscosities of $\mathrm{ms} 2$ and $\mathrm{ms} 3$ is somewhat larger; the values for both emulsions are indicated in Fig. 3. No significance can be attached to the difference observed. It is still not beyond the experimental error, which is larger for the steady-state (a few percent) than it is for the dynamic viscosities (less than $2 \%$ ). Also for ms 2 and ms 3 we conclude that two or more relaxation mechanisms operate. The arguments used are the same as in the case of ms 1 . The computer fits were made on the basis of the same assumptions as those used for ms 1 . For both fits the sum of the squares of the deviations between the best fit and the 64 experimental points was $1.6 \times 10^{-2}$. A survey of the model parameters is again given in Table III.

In Fig. 4 the dynamic viscosities of the microemulsions mc 1 and mc 2 relative to that of water are shown for $T=20.0^{\circ} \mathrm{C}$ and $T=24.0^{\circ} \mathrm{C}$, respectively. These measurements show a maximum in $\eta_{2}$, and in $\eta_{1}$ a transition between two plateau values. This can be concluded on the basis of behavior of $\eta_{1}$ and $\eta_{2}$ as a function of frequency, given by general linear viscoelasticity. The char-

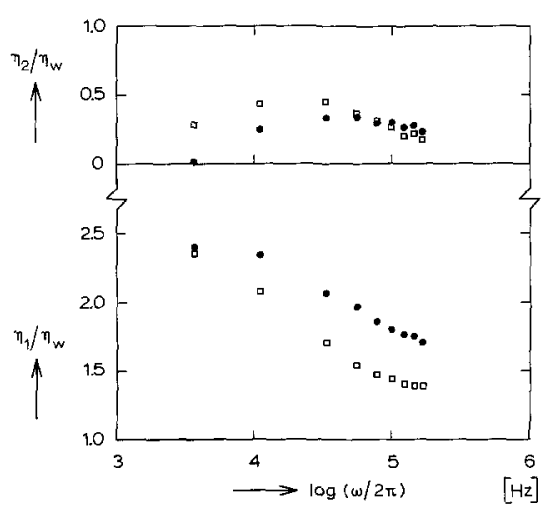

FIG. 4. Measured dynamic viscosity of the microemulsion $\mathrm{mc} 1$ at $20.0^{\circ} \mathrm{C}$ and $\mathrm{mc} 2$ at $24.0^{\circ} \mathrm{C}$ as a function of frequency. Filled circles, mc 1. Open squares, me 2 . 
acteristic times pertinent to the maxima are: $t=5 \mu \mathrm{sec}$ for $\mathrm{mc} 1$ and $t \simeq 7 \mu \mathrm{sec}$ for $\mathrm{mc} 2$.

In Fig. 5 the dynamic viscosity of mc 3 relative to that of water is shown for $T$ $=15.0^{\circ} \mathrm{C}$ and $T=16.0^{\circ} \mathrm{C}$. It appears that the characteristic time $t$ pertinent to the position of the maximum in $\eta_{2}$ increases with temperature. The maximum itself, however, is too broad to be caused by one relaxation mechanism only. Conclusions from Fig. 5 will be drawn in the Discussion.

\section{DISCUSSION}

For the interpretation of the measurements we will consider three possible causes of the elasticity displayed by the systems we investigated. These causes are:

(a) arrangements of surfactant molecules, such as assemblies of rods, lamellae, and spheres;

(b) electrical charges on the emulsion droplets;

(c) the response to deformation of the individual droplets together with their surfactant shell.

The mesoemulsions were somewhat turbid, which might be due to structures meant sub a. Their steady-state viscosities, however, were independent of the shear rate between $10^{-2}$ and $10^{+2} \mathrm{sec}^{-1}$ and they were relatively small. We therefore concluded that in these systems macroscopic arrangements were absent, which is in agreement with the investigations $(5,6)$ of dielectrical properties, viscosity, and turbidity of similar emulsions.

The microemulsion systems were more translucent, had a uniform bluish-white appearance if observed perpendicular to incident light and a yellowish appearance if observed in transmitted light. In agreement with Shinoda and Kunieda (8) these systems will be considered to be oil-swollen micellar solutions.

Since only nonionic surfactants were used for both the ms and the mc systems,

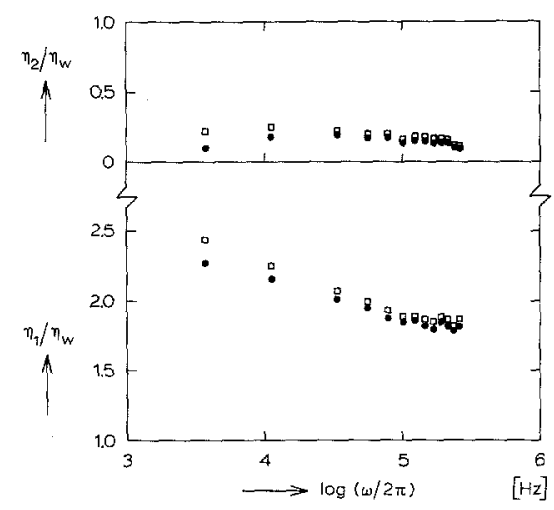

FIG. 5. Measured dynamic viscosity of the microemulsion mc 3 as a function of frequency. Open squares, $16.0^{\circ} \mathrm{C}$. Filled circles, $15.0^{\circ} \mathrm{C}$.

it may be assumed that the surface potential of the droplets was small. Nevertheless, the electrical interactions between emulsion droplets may not be negligible, because the ionic strength in the systems investigated was low. Hence, we cannot exclude the formation of a crystal-like lattice of droplets due to repulsive forces, which may provide an emulsion with elasticity. Such lattices can be recognized from their iridescence, as was demonstrated for lattices in aqueous suspensions of charged spherical polymer particles (14) formed when the ionic strength was extremely low. However, no such phenomenon was observed in our systems. Less pronounced effects of droplet charges screened by a diffuse double layer are viscoelasticity due to the deformation of the double layer, the " primary effect" (15), and viscoelasticity due to binary particle interactions, being the "secondary effect" (16). From the absence of dielectrical dispersion in the measurements of Clause et al. (6) it may be concluded that the mesoemulsions studied do not show a primary electroviscoelastic effect for frequencies up to 5 $\mathrm{MHz}$. Theory on the secondary electroviscoelastic effect for systems with a low ionic strength is not yet available.

So there is no evidence for structures (cause a) or electrical charges (cause b) to provide the mechanisms responsible for the 
observed viscoelasticity, although we cannot exclude them. However, there might be an indication that droplet deformation does. From an estimation of model parameters this mechanism can indeed be expectedin the system studied-to make itself apparent in the frequency range investigated.

Considering therefore for the time being droplet deformation as the active mechanism, we note that the interpretation of the measurements using models $\mathrm{A}$ and $\mathrm{B}$ of (1) leads to consistent sets of parameter values. The values obtained for $\kappa$ are very small compared with those for the surface elasticities found near the CMC $(17,18)$ for nonionic surfactants at air-water interfaces $\left(E_{0} \simeq 60 \mathrm{mN} \mathrm{m}^{-1}\right.$ ). Exchange of surfactant between droplet interfaces and bulk phases containing monomers may, however, reduce the dynamic area elasticity. An even more drastic reduction is to be expected (18) if micelles with a very fast micellization rate are present in the bulk phases. Both $\kappa$ and $\sigma$ are then functions of frequency. This results in a flattening of the relaxation peak that corresponds to $\kappa$. If the peaks that correspond to $\kappa$ and $\gamma$, respectively, are sufficiently apart in frequency, the $\gamma$-peak may not be affected at all and the analysis of the previous section will still give correct values for $\gamma$ and $\zeta$.

In the fit with model $B$ we used one fixed parameter for which a good estimation is possible, viz. the film thickness $d$. The values found for $\gamma+\gamma^{\prime}$ and $\eta^{\prime \prime}$ make sense and are in agreement with those found for $\gamma$ and $\zeta / a$ using model $\mathrm{A}$, but the physical basis of applying model B in this case is weak. In particular, it was made plausible in the previous section that the interfacial film might be monomolecular. In that case the use of independent interfacial tensions on the two sides of the film in model $B$ is questionable.

If the characteristic times found for the microemulsions mc 1 and mc 2 are linked with the interfacial tension of sperical droplets, their order of magnitude is $\lambda \simeq a \eta / \gamma$. A value for the droplet radius can be esti- mated by assuming a monomolecular coverage of the interfaces. If the area per adsorbed molecule is $0.5 \mathrm{~nm}^{2}$ for $\mathrm{mc} 1$ (and $0.7 \mathrm{~nm}^{2}$ for mc 2) - see (19) - the amount of adsorbed surfactant is $4 \mathrm{~g} / \mathrm{dl}$ (and $2 \mathrm{~g} / \mathrm{dl}$ ), and the molecular weight of the surfactant is 600 (and 640), we arrive at $a \simeq 30 \mathrm{~nm}$ for $\mathrm{mc} 1$ (and $a \simeq 60$ $\mathrm{nm}$ for mc 2). In both bases $\gamma \simeq 10 \mu \mathrm{N} \mathrm{m}^{-1}$. These values for $\gamma$ and $a$ are in agreement with theories $(20,21)$ about microemulsions prepared with nonionic surfactants.

If the maximum of $\eta_{2}$ in Fig. 5 corresponds to the interfacial-tension relaxation mechanism, its shift with temperature indicates that the value of $a / \gamma$ increases with temperature. Such a tendency is in agreement with observations of Shinoda et al. (8) and with the theoretical considerations of Robbins (20) and Reiss (21) for microemulsions with nonionic surfactants. The observations of Shinoda et al. suggest an increase of the droplet radii when temperature is raised. It is generally accepted that this effect is caused by the dehydration of surfactant molecules (20), resulting in shrinkage of the polar region of the interfacial film, which leads to an increase in the radius of curvature of the film. According to theory larger droplets have smaller interfacial tensions $(20,21)$.

As mentioned in the previous section, the flattened maxima in Fig. 5 indicate that there is a distribution of relaxation times; an obvious explanation is that the distribution of $a / \gamma$ values is not extremely sharp.

The presented experimental results and their analyses, in particular those concerning the microemulsions, have a preliminary character. Further research will particularly be aimed at the distinction between electroviscoelastic effects and droplet-deformation effects. In the future an improved model will be needed which will integrate the essentials of our models A and B.

\section{APPENDIX I: NOTATION}

(Outer) radius of emulsion droplets Molecular area 


$\begin{array}{ll}c & \text { Surfactant concentration } \\ d & \text { Thickness of interfacial layer } \\ E_{0} & \text { Surface elasticity } \\ f & \text { Correction factor } \\ i & =\sqrt{-1} \\ M & \text { Molecular weight } \\ N_{\mathrm{A}} & \text { Avogadro's number } \\ R & =f \phi^{-1 / 3} \\ S & \text { Sedimentation coefficient } \\ t & \text { Characteristic time } \\ T & \text { Temperature } \\ \gamma, \gamma^{\prime} & \text { Interfacial tensions } \\ \zeta & \text { Dynamic surface-shear viscosity } \\ \eta & \text { Viscosity } \\ \eta^{*} & =\eta_{1}-i \eta_{2}, \text { complex emulsion vis- } \\ & \quad \text { cosity } \\ \bar{\eta} & \text { Steady-state viscosity } \\ \eta^{\prime} & \text { Droplet viscosity } \\ \eta^{\prime \prime} & \text { Interfacial viscosity } \\ \kappa & \text { Dynamic area elasticity } \\ \lambda & \text { Relaxation time } \\ \mu & \text { Dynamic surface-shear elasticity } \\ \nu & \text { Frequency } \\ \rho & \text { Density } \\ \sigma & \text { Dynamic area viscosity } \\ \phi & \text { Particle volume fraction } \\ \omega & \text { Angular frequency } \\ & \end{array}$

$\begin{array}{ll} & \text { Subscripts } \\ \text { B } & \text { Benzene } \\ \text { D } & \text { Disperse phase } \\ \text { 0 } & \text { Continuous phase } \\ \text { W } & \text { Water }\end{array}$

\section{ACKNOWLEDGMENTS}

The authors are grateful to Dr. A. Capelle and Drs. W. Jansen of the Chemische Fabriek Servo BV (Delden) for their support in emulsion and surfactant technology. Thanks are due to Mr. H. A. J. M. Bevers for the chemi- cal analyses and to Prof. P. F. Mijnlieff for his valuable discussions of this paper.

\section{REFERENCES}

1. Oosterbroek, M., and Mellema, J., J. Colloid Interface Sci., in press.

2. Waterman, H. A., Oosterbroek, M., Beukema, G. J., and Altena, E. G., Rheol. Acta 18, 585 (1979).

3. Oosterbroek, M., Waterman, H. A., Wiseall, S. S., Altena, E. G., Mellema, J., and Kip, G. A. M., Rheol. Acta 19, 497 (1980).

4. Oosterbroek, M., Tropper, R. B., and Mellema, J., Rheol. Acta 19, 203 (1980).

5. Matsumoto, S., and Sherman, P., J. Colloid Interface Sci. 30, 525 (1969).

6. Clause, M., Sherman, P., and Sheppard, R. J., J. Colloid Interface Sci. 56, 123 (1976).

7. Burgers, J. M., Proc. K. Ned. Akad. Wet. 44, 105 (1941).

8. Shinoda, K., and Kunieda, H., in "Microemulsions, Theory and Practice'” (L. M. Prince, Ed.), p. 57. Academic Press, New York, 1977.

9. Simha, R., J. Appl. Phys. 23, 1020 (1952).

10. Thomas, D. G., J. Colloid Sci. 20, 267 (1965).

11. Krieger, I. M., Adv. Colloid Interface Sci. 3, 111 (1972).

12. Mittal, K. L., J. Pharm. Sci. 61, 1335 (1972).

13. Rösch, M., in "Nonionic Surfactants" (M. J. Schick, Ed.), p. 753. Marcel Dekker, New York, 1966.

14. Williams, R., Crandall, R. S., and Wojtowicz, P. J., Phys. Rev. Lett. 37, 348 (1976).

15. Russel, W. B., J. Fluid Mech. 85, 673 (1978).

16. Russel, W. B., J. Fluid Mech. 85, 209 (1978).

17. Lucassen, J., and Giles, D., J. Chem. Soc., Faraday Trans. I 71, 217 (1975).

18. Lucassen, J., Faraday Discuss. Chem. Soc. 59, 76 (1975).

19. Lange, H., in "Nonionic Surfactants" (M. J. Schick, Ed.), p. 443. Marcel Dekker, New York, 1966.

20. Robbins, M. L., in "Micellization, Solubilization, and Microemulsions" (K. L. Mittal, Ed.), Vol. 2, p. 713. Plenum Press, New York, 1977.

21. Reiss, H., J. Colloid Interface Sci. 53, 61 (1975). 